\title{
SOME CONSIDERATIONS ON BERTH PRODUCTIVITY REFERRED ON DRY BULK CARGOES IN A MULTIPURPOSE SEAPORT
}

\author{
Deda Đelović, Dijana Medenica Mitrović
}

Preliminary communication

Productivity at the berth is one from the group of port productivity measures which is closely tied to ship turnaround times. Gross berth productivity between a ship's arrival and departure from berth is among the broadest definitions of productivity. Net berth productivity is value of productivity related to the operational time at the berth. Based on the recognized facts from available literature about minor share of researches related to the berth productivity in the handling process with dry bulk cargoes in a multipurpose seaport, it was decided to take this port productivity metrics as an object of a research Concrete results shown in this paper are related to the Dry Bulk Cargo Terminal in the Port of Bar. By the consideration done in this paper is confirmed starting hypothesis about significant differences between gross and net berth productivity (about significant share of nonoperational time in the total berthing time).

Keywords: berth; productivity; ship turnaround time

Neki aspekti analize produktivnosti pri pretovaru suhih rasutih tereta u višenamjenskoj morskoj luci

Prethodno priopćenje

Produktivnost na vezu je jedno od mjerila produktivnosti u procesu lučkog pretovara koje je direktno povezano s vremenom opsluživanja broda u luci. Bruto produktivnost na vezu, koja se odnosi na ukupno vrijeme boravka broda na vezu, spada u grupu najopćijih definicija produktivnosti. Neto produktivnost na vezu se odnosi na dio vremena boravka broda na vezu tijekom kojeg se provode pretovarne operacije. Osnovni motiv za pokretanje istraživanja čiji su rezultati prikazani u članku je činjenica, utvrđena analizom dostupne literature, da je učešće razmatranja produktivnosti pri pretovaru suhih rasutih tereta u viešnamjenskim morskim lukama na jako niskoj razini. Dominiraju istraživanja koja se odnose na produktivnot u procesu pretovara kontejnera. U članku prezentirani rezultati se odnose na Terminal za suhe rasute terete u Luci Bar. Razmatranjima koja su sprovedena je potvrđena polazna hipoteza istraživanja o postojanju značajnih razlika između razine bruto i neto produktivnosti na vezu (o značajnom učešću neoperativnog vremena u ukupnom vremenu boravka broda na vezu).

Ključne riječi: produktivnost; vez; vrijeme opsluživanja broda

\section{Introduction}

Ports form a vital link in the overall trading chain [1]. In fact, a meaningful evaluation of a port's performance will require sets of measures (port performance cannot be determined by only one indicator or by a single allencompassing value) relating to: the duration of ship's stay in port; the quality of the cargo handling; the quality of service to inland transport vehicles during their passage through the port [2].

Traditionally, the performance of ports has been variously evaluated by calculating cargo-handling productivity at berth, by measuring a single factor productivity or by comparing actual with optimum throughput over a specific time period. In more recent years, significant progress has been made concerning the measurement of efficiency in relation to productive activities. Two more complex, yet more appropriately holistic approaches, named Data Envelopment Analysis (DEA) and Stochastic Frontier Analysis (SFA), have been increasingly utilized to analyze port production and performance [1]. Ports use performance metrics to monitor their performances, plan capital expenditures, project revenue, etc. [3]. Perhaps of more direct concern to port management than financial indicators are operational ones. Through the control of operational performance, management will control the financial performance of the port as well [4].

Productivity and efficiency are the two important concepts and are frequently utilized to measure port performance [5].
Port efficiency is an important factor to stimulate port competitiveness and boost regional development and is often associated with productivity [6]. Ports cannot meet carrier needs for productivity without technology, and they cannot achieve the required financial results without improving efficiency [7].

Productivity can most usefully be defined as the combined result of resource utilization and operational efficiency. Productivity of a given asset may be increased either by increasing utilization or by increasing operating efficiency. Using cranes as an example, crane productivity could be increased by operating cranes more hours per day (utilization) or by achieving more lifts per operating hour (efficiency) [3].

Productivity is defined as the amount of output per unit of input $[5,8,9]$ and is based on local circumstances of labor, equipment, land, capital, management, infrastructure and politics $[1,7]$. It can be also defined as a summary measure of a quantity and quality work of performance with resource utilization considered [10].

In the available literature sources can be found numerous additional definitions of productivity.

For example, at the operational level, productivity is defined as the average of the gross moves per hour for each ship call recorded during a year. Gross moves per hour for a single vessel call is defined as the total container moves (on-load, off-load and repositioning) divided by the number of hours for which the vessel is at berth [11].

There are seven different productivity measures which port terminal operators need to compute, although they may wish to include others for monitoring their 
productivity. These core productivity measures are: Ship productivity; Crane productivity; Quay productivity; Terminal area productivity; Equipment productivity; Labor productivity; Cost effectiveness; [1].

In general, the measurement of port productivity has been greatly impeded by the following factors: the number of parameters involved; The lack of up-to-date, factual and reliable data, collected in an accepted manner and available for dissemination; the absence of generally agreed and acceptable definitions; the profound influence of local factors on the data obtained; the divergent interpretation given by various interests to identical results; [5].

Very detailed literature reviews related to the productivity in ports are presented in $[1,3,5,9]$. As concluded in [9], over the last years there has been a proliferation of research which investigates the efficiency of ports and terminals and majority of published works has been applied to the container port sector.

Criteria for useful productivity measures might include: Comparability - The chosen measures should reflect aspects of port and terminal performance that can reliably be compared across coastal and national geographies; Accuracy - The measures should be derived through straightforward analysis of reliable, available data; Replicability - The effects of year-to-year variations in exogenous factors such as rail industry performance or weather should be noted, and ideally it should be possible to correct for such variations; Relevance - The measures should document factors that will enter into operational choices, capital investments, and cargo routing decisions [3].
There are four approaches in measuring port productivity. The first approach of measuring port productivity can be summarised as short- and long-term categories. In the short term, there are four distinct areas that lend themselves to quantification: the stevedoring process, gate cycles, intermodal cycles and yard operations. The long-run concerns, on the other hand, are overall throughput, terminal throughput density, berth throughput density and container storage dwell time. The second approach outlines several indicators of productivity: port accessibility, gross berth productivity, net berth productivity, gross gang productivity, net gang productivity. The third approach to measuring port productivity can be divided into three parts: stevedoring productivity, waterfront reliability and stevedoring reliability. Finally, the fourth approach is based on the assumption that port production can be divided into categories of seaside, marshalling yard and landside [5].

Productivity at the berth is one from the group of port productivity measures which is closely tied to ship turnaround times. It shows which ports and terminals are the best at working ships and getting them back to sea quickly. Gross berth productivity between a ship's arrival and departure from berth, with no adjustments for labor or equipment down time regardless of the reason - is among the broadest definitions of productivity [12]. Net berth productivity is value of productivity related to the operational time at the berth (when nonoperational time is deducted from total berthing time).

One of the most significant indicators of service quality to ship operators is ship turnaround time. Its structure is shown by the next diagram (Fig. 1) [13].

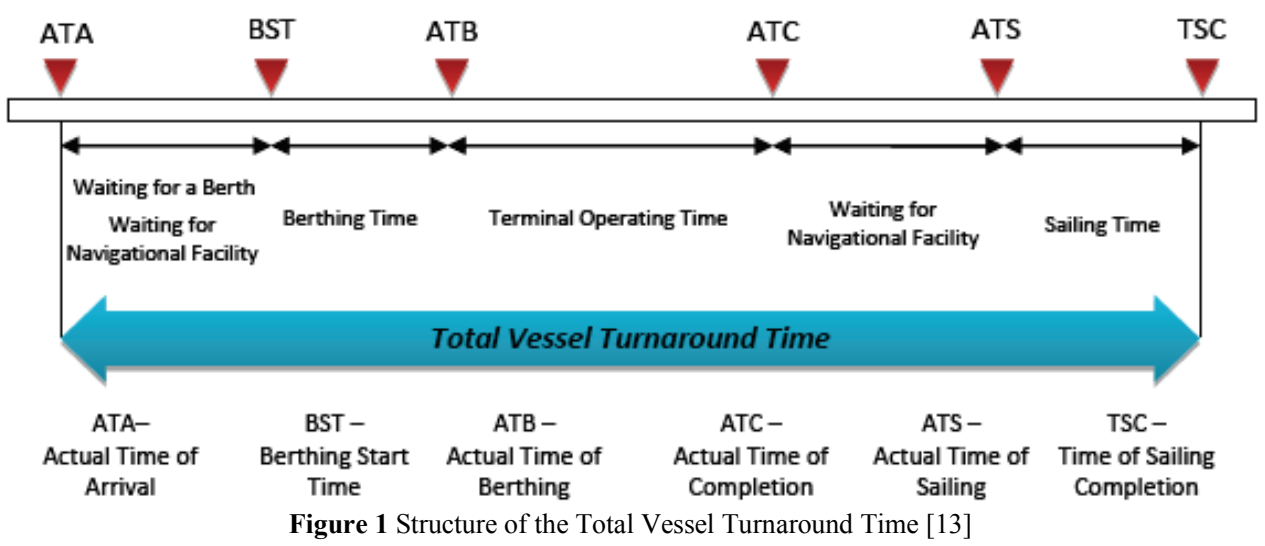

It is obvious that most of principal common objectives of both key parties (ship/carrier and terminal operator) in the port cargo handling process are related to productivity: ship/carrier - maximize productivity on the ship, minimize port time; terminal operator - maximize berth productivity, minimize berth occupancy; [14].

Increased berth productivity lead to reduction of the Terminal Operating Time (length of the ship s time in a port - sum of operational and non operational periods of time at berth).

In general, it is vital to evaluate port performance in relation to how efficient are their services from the perspective of the port users: ship-owners, shippers (importers and exporters), land transport owners, etc. [9].

Based on the recognized facts from the available literature about minor share of researches related to the berth productivity in the handling process with dry bulk cargoes in the multipurpose seaports, it was decided to take this port productivity metrics as an object of a research in this paper. The key objective of research conducted is to analyze values of the berth productivity (gross and net) with different dry bulk cargoes and then to identify and systematize influential factors which determine values of the berth productivity in a multipurpose seaport. Starting hypothesis of the research is: there are significant differences between gross and net berth productivity - there is a significant share of nonoperational time in the total berthing time.

Concrete results shown in this paper are related to the Dry Bulk Cargo Terminal in the Port of Bar. 


\section{An analysis of values of berth productivity at the Dry bulk cargo terminal in a multipurpose seaport \\ 2.1 Description of the Port of Bar as a multipurpose} seaport

Using principle elements of the WORKPORT model structure [15], in Table 1 are systematized basic performances of the Port of Bar [16].
Some from the group of basic operational features of the Dry Bulk Cargo Terminal in Port of Bar, with special focus on the port machinery at operational quay (berths), are systematized in Table 2.

Table 1 Basic performances of The Port of Bar

\begin{tabular}{|c|c|}
\hline Element & Year 2014 \\
\hline $\begin{array}{l}\text { Ownership/ } \\
\text { Management } \\
\text { Model }\end{array}$ & $\begin{array}{l}\text { The Port of Bar is a landlord port; it is functioning based on the Montenegrin Law on ports; at the port area are operating } \\
\text { two main Port Terminal Operators: Port of Bar H. Co. (a share holding company, where majority of shares }-54 \%-\text { are } \\
\text { owned by the Government of Montengro) and the Port of Adria, where major part of shares }-62 \%-\text { are owned by the } \\
\text { Turkish Company Global Ports; } \\
\text { The first Port Terminal Operator, Port of Bar H. Co., is managing following specialized terminals: Terminal for dry bulk } \\
\text { cargoes, Terminal for liquid cargoes, Terminal for grain, Ro-Ro and Passenger terminal; } \\
\text { The second Port Terminal Operator, Port of Adria, is managing Terminal for general cargoes, Terminal for containers and } \\
\text { Terminal for sawn timber; }\end{array}$ \\
\hline Throughput Structure & $\begin{array}{l}\text { Main cargo groups which are handled in The Port of Bar are: liquid bulk cargoes (LB); dry bulk cargoes (DB); general } \\
\text { Lo-Lo cargoes (G-Lo-Lo); Containers Lo-Lo (C); General Ro-Ro cargoes (G-Ro-Ro); }\end{array}$ \\
\hline $\begin{array}{l}\text { Cargo Handling } \\
\text { Technologies }\end{array}$ & $\begin{array}{l}\text { Handling operations with general cargoes are mechanized, as well as handling operations with dry bulk cargoes; } \\
\text { Operations with liquid cargoes are highly mechanized and automatized; }\end{array}$ \\
\hline Information System & $\begin{array}{l}\text { Automatized integral information system which covers all business activities; Internet; Intranet; EDI system is } \\
\text { implemented in process of distribution of Quality system documentation; }\end{array}$ \\
\hline $\begin{array}{l}\text { Work Force/Work } \\
\text { Organization/ } \\
\text { Employment } \\
\text { Relations/Labour Relations }\end{array}$ & $\begin{array}{l}\text { Hierarchical work organization; Degree of work force specialization is increased; Greater emphasis on qulity aspect of } \\
\text { provided services; Internationally certified Quality Management System, modeled according to standard ISO 9001: 2008, } \\
\text { exists. }\end{array}$ \\
\hline $\begin{array}{l}\text { Port Function/ } \\
\text { Port Development }\end{array}$ & $\begin{array}{l}\text { Handling operations are in the focus; The Port of Bar, at complete territory (expcept a part of Ro-Ro and Passenger } \\
\text { terminal), is a Free Zone; }\end{array}$ \\
\hline $\begin{array}{l}\text { Health And Safety Aspect } \\
\text { Of Working Environment }\end{array}$ & $\begin{array}{l}\text { Improved training in safety awareness; Decreasing accidents rate and physical health problems; Health and safety policy } \\
\text { exists; } \\
\text { Complete port territory is under video surveillance; ISPS Code is fully implemented since July } 1 \text { st, 2004; }\end{array}$ \\
\hline Environmental Protection & $\begin{array}{l}\text { Process of introducing certified Environmental Management System is taking place; Analyses of environmental aspects } \\
\text { are obligatory part of all projects realized within the port area; }\end{array}$ \\
\hline
\end{tabular}

Table 2 Operational features of the Dry Bulk Cargo Terminal in the Port of Bar

\begin{tabular}{|c|c|c|c|c|c|c|c|c|c|c|}
\hline \multirow[t]{2}{*}{ Berth } & \multirow[t]{2}{*}{$\begin{array}{l}\text { Water } \\
\text { depth } \\
(\mathrm{m})\end{array}$} & \multicolumn{2}{|c|}{$\begin{array}{l}\text { Availability of the } \\
\text { shore port } \\
\text { machinery }\end{array}$} & \multirow[t]{2}{*}{$\begin{array}{l}\text { Type of the port } \\
\text { machinery }\end{array}$} & \multicolumn{5}{|c|}{$\begin{array}{l}\text { Cargo types which can be handled } \\
\text { (LB - Liquid bulks; DB - Dry bulks; G - } \\
\text { Lo-Lo - General Lo-Lo; C - containers Lo- } \\
\text { Lo; G - Ro-Ro - General Ro-Ro; }\end{array}$} & \multirow{2}{*}{$\begin{array}{l}\text { Cargo handling operations } \\
\text { (sum of possible handling } \\
\text { operations) }\end{array}$} \\
\hline & & Yes & No & & LB & DB & $\begin{array}{c}\text { G } \\
\text { Lo-Lo }\end{array}$ & $\mathrm{C}$ & $\begin{array}{c}\mathrm{G} \\
\text { Ro-Ro }\end{array}$ & \\
\hline$(1)$ & (2) & (3)-1 & $(3)-2$ & (4) & $(5)-1$ & $(5)-2$ & $(5)-3$ & $(5)-4$ & $(5)-5$ & $(6)$ \\
\hline SO1 & 6,5 & & $*$ & - & & $*$ & & & & Dry bulks: Ship - silo (1); \\
\hline 03 & 14,0 & * & & \multirow[t]{3}{*}{$\begin{array}{l}\text { Ship to shore gantry } \\
\text { cranes ( } 3 \text { pcs.), SWL } 12 \\
\text { t, rail mounted, } \\
\text { movable by all three } \\
\text { berths; }\end{array}$} & $*$ & $*$ & * & & & $\begin{array}{l}\text { Liquid bulks: Ship - reservoir (1); } \\
\text { Dry bulks: Ship to shore/ truck/ } \\
\text { wagon (and vice versa) (3); } \\
\text { General Lo-Lo: ship to } \\
\text { shore/truck/ wagon (and vice } \\
\text { versa) (3); }\end{array}$ \\
\hline 02 & 14,0 & $*$ & & & & $*$ & $*$ & & & \multirow[b]{2}{*}{$\begin{array}{l}\text { Dry bulks: Ship to shore/truck/ } \\
\text { wagon (and vice versa); ship to } \\
\text { silo (and vice versa) (4); } \\
\text { General Lo-Lo: ship to } \\
\text { shore/truck/ wagon (and vice } \\
\text { versa) (3); }\end{array}$} \\
\hline 01 & 14,0 & * & & & & $*$ & $*$ & & & \\
\hline
\end{tabular}

\subsection{Methodology of the berth productivity analysis}

Gross berth productivity, $P_{\mathrm{gb}}(\mathrm{t} / \mathrm{h})$, per cargo, is calculated using the following equation:

$P_{\mathrm{gb}}=\sum$ (total cargo quantity loaded/unloaded in/from ships in a year)(t)/ $\sum$ (total berthing time of all ships in a year) (h)

Net berth productivity, $P_{\mathrm{nb}}(\mathrm{t} / \mathrm{h})$, per cargo, is calculated using Eq. (2):
$P_{\mathrm{nb}}=\sum$ (total cargo quantity loaded/unloaded in/from ships in a year)(t) $/ \sum$ (total operational time of all ships in a year) (h)

In the process of berth productivity analysis are used methods of mathematical statistics [17] as well as a methodology adjusted to the concrete object of the analysis - elements of this methodology are shown in Fig. 2. 


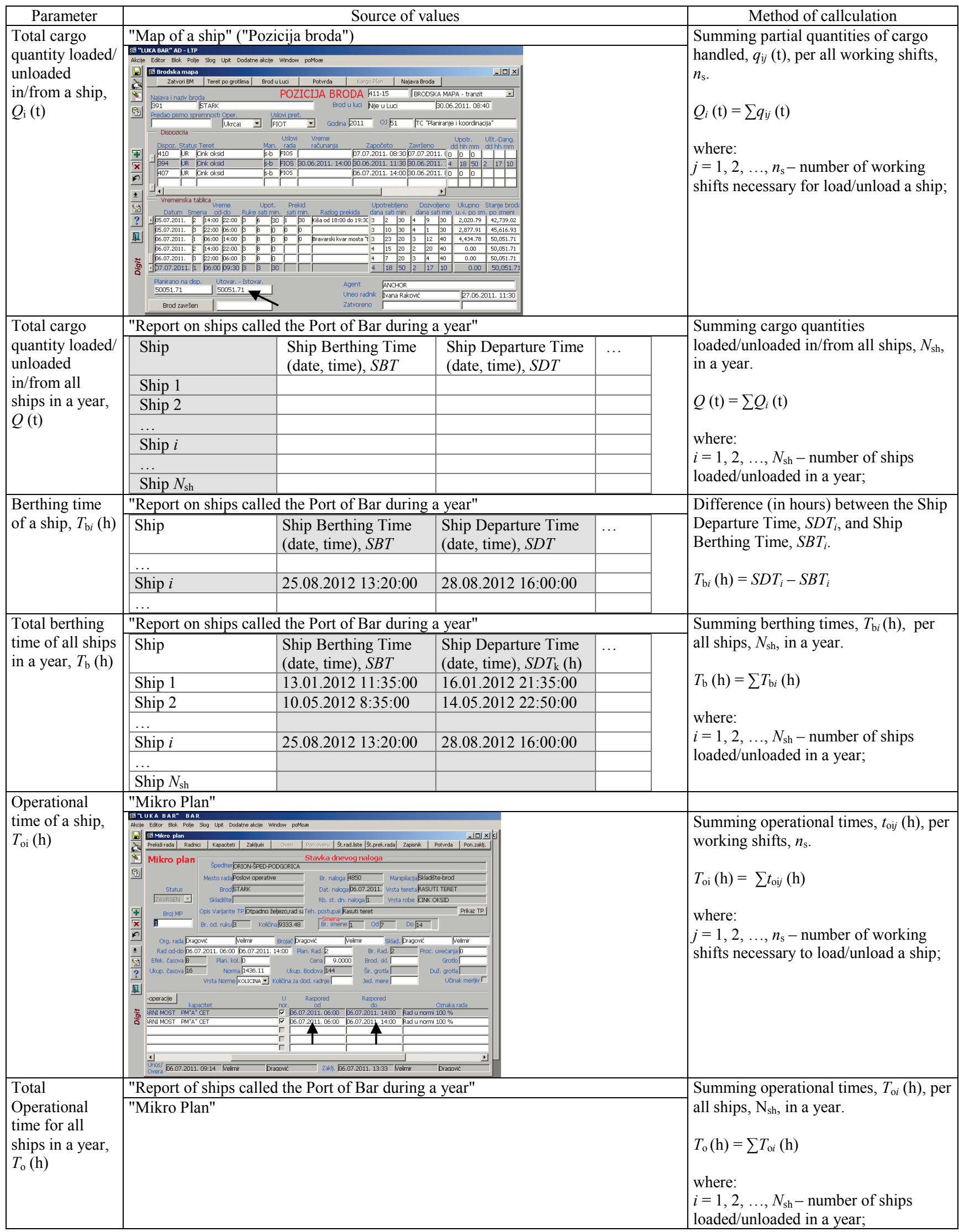

Figure 2 Methodology used in the process of berth productivity analysis

\subsection{Results of the berth productivity analysis}

Using methodology described in the previous chapter (2.2), here are presented results of the gross and net berth productivity at the Dry Bulk Cargo terminal in the Port of Bar from 2010 to 2014 - total number of ship calls analyzed: 581 ; total number of cargo types analyzed: 10 , with the biggest share in the total throughput at this Terminal [16]. 


\subsubsection{Resuts of the grosss berth productivity analysis}

Results of the grosss berth productivity, $P_{\mathrm{gb}}(\mathrm{t} / \mathrm{h})$, analysis are presented in the next table (Tab. 3).

Table 3 Gross berth productivity in the Port of Bar for the period from 2010 to 2014

\begin{tabular}{|c|c|c|c|c|c|c|c|}
\hline \multirow{2}{*}{ Cargo } & \multirow{2}{*}{$\begin{array}{l}\text { Handling } \\
\text { operation }\end{array}$} & \multirow{2}{*}{ Parameter } & \multicolumn{5}{|c|}{ Values of parameters per years } \\
\hline & & & 2010 & 2011 & 2012 & 2013 & 2014 \\
\hline \multirow[t]{3}{*}{ C1: cement } & \multirow[t]{3}{*}{ Ship - Silo } & $\begin{array}{l}\text { Total cargo quantity } \\
\text { loaded/unloaded in/from ships in } \\
\text { a year }(\mathrm{t})\end{array}$ & 114777,3 & 92913,3 & 90962,6 & 94161 & 76112 \\
\hline & & $\begin{array}{l}\text { Total berthing time for all ships } \\
\text { in a year }(\mathrm{h})\end{array}$ & 3172,4 & 2693,4 & 2169,5 & 2527,4 & 2458,6 \\
\hline & & Gross berth productivity $(\mathrm{t} / \mathrm{h})$ & 36,2 & 35,2 & 42,1 & 37,3 & 30,9 \\
\hline \multirow[t]{3}{*}{ C2: Zink ore } & \multirow[t]{3}{*}{$\begin{array}{l}\text { Open storage } \\
\text { area - ship }\end{array}$} & $\begin{array}{l}\text { Total cargo quantity } \\
\text { loaded/unloaded in/from ships in } \\
\text { a year }(\mathrm{t})\end{array}$ & 105650,4 & 149944,4 & 83664,5 & - & 32566,5 \\
\hline & & $\begin{array}{l}\text { Total berthing time for all ships } \\
\text { in a year }(\mathrm{h})\end{array}$ & 491,2 & 676,9 & 266,9 & - & 129,9 \\
\hline & & Gross berth productivity $(\mathrm{t} / \mathrm{h})$ & 215,1 & 221,5 & 313,4 & - & 250,7 \\
\hline \multirow[t]{3}{*}{ C3: Alumina } & \multirow[t]{3}{*}{ Ship - truck } & $\begin{array}{l}\text { Total cargo quantity } \\
\text { loaded/unloaded in/from ships in } \\
\text { a year }(\mathrm{t})\end{array}$ & 7925,2 & 21412,7 & 15500 & 4150,1 & 33655,9 \\
\hline & & $\begin{array}{l}\text { Total berthing time for all ships } \\
\text { in a year }(\mathrm{h})\end{array}$ & 424,4 & 349,7 & 271,8 & 76,6 & 380,8 \\
\hline & & Gross berth productivity $(\mathrm{t} / \mathrm{h})$ & 18,7 & 61,2 & 57 & 54,9 & 88,4 \\
\hline \multirow[t]{3}{*}{$\begin{array}{l}\text { C4: Crashed } \\
\text { stone }\end{array}$} & \multirow[t]{3}{*}{$\begin{array}{l}\text { Open storage } \\
\text { area - ship }\end{array}$} & $\begin{array}{l}\text { Total cargo quantity } \\
\text { loaded/unloaded in/from ships in } \\
\text { a year }(t)\end{array}$ & 112500 & 40430,8 & 15500 & 44350 & 49650 \\
\hline & & $\begin{array}{l}\text { Total berthing time for all ships } \\
\text { in a year }(\mathrm{h})\end{array}$ & 3369,7 & 1407,7 & 156 & 479,2 & 564,3 \\
\hline & & Gross berth productivity $(\mathrm{t} / \mathrm{h})$ & 33,4 & 28,7 & 99,4 & 92,6 & 87,9 \\
\hline \multirow[t]{3}{*}{$\begin{array}{l}\text { C5: Zinc } \\
\text { concentrate }\end{array}$} & \multirow[t]{3}{*}{$\begin{array}{l}\text { Open storage } \\
\text { area - ship }\end{array}$} & $\begin{array}{l}\text { Total cargo quantity } \\
\text { loaded/unloaded in/from ships in } \\
\text { a year }(t)\end{array}$ & 2792,9 & 3564,7 & 2875 & 9503,9 & - \\
\hline & & $\begin{array}{l}\text { Total berthing time for all ships } \\
\text { in a year }(\mathrm{h})\end{array}$ & 34,2 & 112,7 & 28,3 & 98,8 & - \\
\hline & & Gross berth productivity $(\mathrm{t} / \mathrm{h})$ & 81,8 & 31,6 & 101,8 & 96,2 & - \\
\hline \multirow[t]{3}{*}{$\begin{array}{l}\text { C6: Iron } \\
\text { scrap }\end{array}$} & \multirow[t]{3}{*}{$\begin{array}{l}\text { Open storage } \\
\text { area - ship }\end{array}$} & $\begin{array}{l}\text { Total cargo quantity } \\
\text { loaded/unloaded in/from ships in } \\
\text { a year }(\mathrm{t})\end{array}$ & 55576,5 & 17462,2 & 29344,1 & 23795,8 & 14683,6 \\
\hline & & $\begin{array}{l}\text { Total berthing time for all ships } \\
\text { in a year }(\mathrm{h})\end{array}$ & 802,2 & 395,5 & 1018,1 & 1060 & 742,3 \\
\hline & & Gross berth productivity $(\mathrm{t} / \mathrm{h})$ & 69,3 & 44,2 & 28,8 & 22,4 & 19,8 \\
\hline \multirow[t]{3}{*}{ C7: Grain } & \multirow[t]{3}{*}{ Silo-ship } & $\begin{array}{l}\text { Total cargo quantity } \\
\text { loaded/unloaded in/from ships in } \\
\text { a year }(t)\end{array}$ & 5458,4 & 8670,5 & 8517,9 & 20359,4 & 9310,1 \\
\hline & & $\begin{array}{l}\text { Total berthing time for all ships } \\
\text { in a year }(\mathrm{h})\end{array}$ & 36,7 & 155,8 & 108,8 & 145 & 95,7 \\
\hline & & Gross berth productivity $(\mathrm{t} / \mathrm{h})$ & 148,9 & 55,7 & 78,3 & 140,4 & 97,3 \\
\hline \multirow[t]{3}{*}{ C8: Salt } & \multirow[t]{3}{*}{ Ship - truck } & $\begin{array}{l}\text { Total cargo quantity } \\
\text { loaded/unloaded in/from ships in } \\
\text { a year }(\mathrm{t})\end{array}$ & 3950 & 10150 & 2650 & 5684,1 & 4800 \\
\hline & & $\begin{array}{l}\text { Total berthing time for all ships } \\
\text { in a year }(\mathrm{h})\end{array}$ & 241,4 & 301,1 & 56,5 & 109,1 & 59,8 \\
\hline & & Gross berth productivity $(\mathrm{t} / \mathrm{h})$ & 16,4 & 33,7 & 46,9 & 52,1 & 80,2 \\
\hline \multirow[t]{3}{*}{ C9: Corn } & \multirow[t]{3}{*}{ Silo - ship } & $\begin{array}{l}\text { Total cargo quantity } \\
\text { loaded/unloaded in/from ships in } \\
\text { a year }(\mathrm{t})\end{array}$ & - & 21644 & 34692 & 2955,3 & 9723,1 \\
\hline & & $\begin{array}{l}\text { Total berthing time for all ships } \\
\text { in a year }(\mathrm{h})\end{array}$ & - & 202,4 & 365,5 & 76,7 & 90,1 \\
\hline & & Gross berth productivity $(\mathrm{t} / \mathrm{h})$ & - & 106,9 & 94,9 & 39,1 & 107,9 \\
\hline \multirow[t]{3}{*}{ C10: Coal } & \multirow[t]{3}{*}{ Ship - truck } & $\begin{array}{l}\text { Total cargo quantity } \\
\text { loaded/unloaded in/from ships in } \\
\text { a year }(t)\end{array}$ & - & 14299 & 20966,8 & 3183,4 & 4899,9 \\
\hline & & $\begin{array}{l}\text { Total berthing time for all ships } \\
\text { in a year }(\mathrm{h})\end{array}$ & - & 129,9 & 197,9 & 32,5 & 36,9 \\
\hline & & Gross berth productivity $(\mathrm{t} / \mathrm{h})$ & - & 110,1 & 105,9 & 97,9 & 132,7 \\
\hline
\end{tabular}

Based on the results given in the previous table, it can be concluded that values of gross berth productivity vary in a very wide range over the analyzed period. These variations are shown in Tab. 4. 
Table 4 Variation of gross berth productivity

\begin{tabular}{|c|c|c|c|c|}
\hline \multirow{2}{*}{ Cargo } & \multirow{2}{*}{ Handling operation } & \multicolumn{3}{|c|}{ Variations of the gross berth productivity } \\
\hline & & Minimal & Maximal & Maximal/minimal \\
\hline C1: cement & Ship - Silo & 30,9 & 42,1 & 1,36 \\
\hline C2: Zink ore & Open storage area - ship & 215,1 & 313,4 & 1,46 \\
\hline C3: Alumina & Ship - truck & 18,7 & 88,4 & 4,73 \\
\hline C4: Crashed stone & Open storage area - ship & 28,7 & 99,4 & 3,46 \\
\hline C5: Zinc concentrate & Open storage area - ship & 31,6 & 101,8 & 3,22 \\
\hline C6: Iron scrap & Open storage area - ship & 19,8 & 69,3 & 3,50 \\
\hline C7: Grain & Silo - ship & 55,7 & 148,9 & 2,67 \\
\hline C8: Salt & Ship - truck & 16,4 & 80,2 & 4,89 \\
\hline C9: Corn & Silo - ship & 39,1 & 107,9 & 2,76 \\
\hline C10: Coal & Ship - truck & 97,9 & 132,7 & 1,36 \\
\hline
\end{tabular}

\subsubsection{Results of the net berth productivity analysis}

In Tab. 5 are presented results of analyses of Net

berth productivity, $P_{\mathrm{nb}}(\mathrm{t} / \mathrm{h})$.

Variations of net berth productivity values are given in Tab. 6.

Table 5 Net berth productivity in the Port of Bar for the period from 2010 to 2014

\begin{tabular}{|c|c|c|c|c|c|c|c|}
\hline \multirow[t]{2}{*}{ Cargo } & \multirow{2}{*}{$\begin{array}{l}\text { Handling } \\
\text { operation }\end{array}$} & \multirow[t]{2}{*}{ Parameter } & \multicolumn{5}{|c|}{ Values of parameters per years } \\
\hline & & & 2010 & 2011 & 2012 & 2013 & 2014 \\
\hline \multirow[t]{3}{*}{ C1: cement } & \multirow[t]{3}{*}{ Ship - Silo } & $\begin{array}{l}\text { Total cargo quantity loaded/unloaded } \\
\text { in/from ships in a year }(t)\end{array}$ & 114777,3 & 92913,3 & 90962,6 & 94161 & 76112 \\
\hline & & Operational time at berth $(\mathrm{h})$ & 1704,1 & 1406,5 & 1308 & 1260 & 1207 \\
\hline & & Net berth productivity $(\mathrm{t} / \mathrm{h})$ & 67,4 & 66,1 & 69,5 & 74,7 & 63,1 \\
\hline \multirow[t]{3}{*}{$\mathrm{C} 2$ : Zink ore } & \multirow[t]{3}{*}{$\begin{array}{l}\text { Open storage } \\
\text { area - ship }\end{array}$} & $\begin{array}{l}\text { Total cargo quantity loaded/unloaded } \\
\text { in/from ships in a year }(t)\end{array}$ & 105650,4 & 149944,4 & 83664,5 & - & 32566,5 \\
\hline & & Operational time at berth $(\mathrm{h})$ & 204,1 & 329,4 & 179,7 & - & 76,9 \\
\hline & & Net berth productivity $(\mathrm{t} / \mathrm{h})$ & 517,6 & 455,2 & 465,6 & & 423,5 \\
\hline \multirow[t]{3}{*}{ C3: Alumina } & \multirow[t]{3}{*}{ Ship - truck } & $\begin{array}{l}\text { Total cargo quantity loaded/unloaded } \\
\text { in/from ships in a year }(\mathrm{t})\end{array}$ & 7925,2 & 21412,7 & 15500 & 4150,1 & 33655,9 \\
\hline & & Operational time at berth $(\mathrm{h})$ & 119,3 & 286,2 & 166,7 & 52,3 & 281,2 \\
\hline & & Net berth productivity $(\mathrm{t} / \mathrm{h})$ & 66,4 & 74,8 & 93,0 & 79,4 & 119,7 \\
\hline \multirow[t]{3}{*}{$\begin{array}{l}\text { C4: Crashed } \\
\text { stone }\end{array}$} & \multirow[t]{3}{*}{$\begin{array}{l}\text { Open storage } \\
\text { area - ship }\end{array}$} & $\begin{array}{l}\text { Total cargo quantity loaded/unloaded } \\
\text { in/from ships in a year }(\mathrm{t})\end{array}$ & 112500 & 40430,8 & 15500 & 44350 & 49650 \\
\hline & & Operational time at berth (h) & 1029,2 & 397,8 & 77,2 & 278,8 & 335,8 \\
\hline & & Net berth productivity $(\mathrm{t} / \mathrm{h})$ & 109,3 & 101,6 & 200,8 & 159,1 & 147,9 \\
\hline \multirow[t]{3}{*}{$\begin{array}{l}\text { C5: Zinc } \\
\text { concentrate }\end{array}$} & \multirow[t]{3}{*}{$\begin{array}{l}\text { Open storage } \\
\text { area - ship }\end{array}$} & $\begin{array}{l}\text { Total cargo quantity loaded/unloaded } \\
\text { in/from ships in a year }(t)\end{array}$ & 2792,9 & 3564,7 & 2875 & 9503,9 & - \\
\hline & & Operational time at berth (h) & 25,7 & 18,3 & 23,5 & 82,3 & - \\
\hline & & Net berth productivity $(\mathrm{t} / \mathrm{h})$ & 108,7 & 194,8 & 122,3 & 115,5 & \\
\hline \multirow[t]{3}{*}{$\begin{array}{l}\text { C6: Iron } \\
\text { scrap }\end{array}$} & \multirow[t]{3}{*}{$\begin{array}{l}\text { Open storage } \\
\text { area - ship }\end{array}$} & $\begin{array}{l}\text { Total cargo quantity loaded/unloaded } \\
\text { in/from ships in a year }(\mathrm{t})\end{array}$ & 55576,5 & 17462,2 & 29344,1 & 23795,8 & 14683,6 \\
\hline & & Operational time at berth $(\mathrm{h})$ & 422,4 & 199,6 & 254,7 & 195,4 & 108,9 \\
\hline & & Net berth productivity $(\mathrm{t} / \mathrm{h})$ & 131,6 & 87,5 & 115,2 & 121,8 & 134,8 \\
\hline \multirow[t]{3}{*}{ C7: Grain } & \multirow[t]{3}{*}{ Silo - ship } & $\begin{array}{l}\text { Total cargo quantity loaded/unloaded } \\
\text { in/from ships in a year }(\mathrm{t})\end{array}$ & 5458,4 & 8670,5 & 8517,9 & 20359,4 & 9310,1 \\
\hline & & Operational time at berth (h) & 22,1 & 38,3 & 37,4 & 96,8 & 43,5 \\
\hline & & Net berth productivity $(\mathrm{t} / \mathrm{h})$ & 247,0 & 226,4 & 227,8 & 210,3 & 214,0 \\
\hline \multirow[t]{3}{*}{ C8: Salt } & \multirow[t]{3}{*}{ Ship - truck } & $\begin{array}{l}\text { Total cargo quantity loaded/unloaded } \\
\text { in/from ships in a year }(\mathrm{t})\end{array}$ & 3950 & 10150 & 2650 & 5684,1 & 4800 \\
\hline & & Operational time at berth (h) & 38,5 & 90,6 & 25,3 & 37,3 & 36,5 \\
\hline & & Net berth productivity $(\mathrm{t} / \mathrm{h})$ & 102,6 & 112,0 & 104,7 & 152,4 & 131,5 \\
\hline \multirow[t]{3}{*}{ C9: Corn } & \multirow[t]{3}{*}{ Silo-ship } & $\begin{array}{l}\text { Total cargo quantity loaded/unloaded } \\
\text { in/from ships in a year }(\mathrm{t})\end{array}$ & - & 21644 & 34692 & 2955,3 & 9723,1 \\
\hline & & Operational time at berth $(\mathrm{h})$ & - & 118,4 & 152,2 & 17,5 & 50,7 \\
\hline & & Net berth productivity $(\mathrm{t} / \mathrm{h})$ & & 182,8 & 227,9 & 168,9 & 191,8 \\
\hline \multirow[t]{3}{*}{ C10: Coal } & \multirow[t]{3}{*}{ Ship - truck } & $\begin{array}{l}\text { Total cargo quantity loaded/unloaded } \\
\text { in/from ships in a year }(\mathrm{t})\end{array}$ & - & 14299 & 6,8 & 3183,4 &, 9 \\
\hline & & Operational time at berth (h) & - & 74,3 & 141,8 & 28 & 24,9 \\
\hline & & Net berth productivity $(\mathrm{t} / \mathrm{h})$ & & 192,4 & 147,9 & 113,7 & 196,8 \\
\hline
\end{tabular}


Table 6 Variation of net berth productivity

\begin{tabular}{|c|c|c|c|c|}
\hline \multirow[t]{2}{*}{ Cargo } & \multirow[t]{2}{*}{ Handling operation } & \multicolumn{3}{|c|}{ Variations of the net berth productivity } \\
\hline & & Minimal & Maximal & Maximal/minimal \\
\hline C1: cement & Ship - Silo & 63,1 & 74,7 & 1,18 \\
\hline C2: Zink ore & Open storage area - ship & 423,5 & 517,6 & 1,22 \\
\hline C3: Alumina & Ship - truck & 66,4 & 119,7 & 1,80 \\
\hline C4: Crashed stone & Open storage area - ship & 101,6 & 200,8 & 1,98 \\
\hline C5: Zinc concentrate & Open storage area - ship & 108,7 & 194,8 & 1,79 \\
\hline C6: Iron scrap & Open storage area - ship & 87,5 & 134,8 & 1,54 \\
\hline C7: Grain & Silo - ship & 210,3 & 247,0 & 1,17 \\
\hline C8: Salt & Ship - truck & 102,6 & 152,4 & 1,49 \\
\hline C9: Corn & Silo - ship & 168,9 & 227,9 & 1,35 \\
\hline C10: Coal & Ship - truck & 113,7 & 196,8 & 1,73 \\
\hline
\end{tabular}

\subsection{Comparison between the Net berth productivity and the Gross berth productivity}

Comparison between the net berth productivity, $P_{\mathrm{nb}}$ $(\mathrm{t} / \mathrm{h})$, and the gross berth productivity, $P_{\mathrm{gb}}(\mathrm{t} / \mathrm{h})$, is done in Tab. 7, based on results of analyses shown in the previous chapters.

Table 7 Comparison between the net berth productivity, $P_{\mathrm{nb}}(\mathrm{t} / \mathrm{h})$, and the gross berth productivity, $P_{\mathrm{gb}}(\mathrm{t} / \mathrm{h})$

\begin{tabular}{|c|c|c|c|c|c|c|c|c|c|c|c|}
\hline \multirow{3}{*}{ Cargo } & \multirow{3}{*}{$\begin{array}{l}\text { Handling } \\
\text { operation }\end{array}$} & \multicolumn{10}{|c|}{ Gross berth productivity, $P_{\mathrm{gb}}(\mathrm{t} / \mathrm{h})$, and Net berth productivity, $P_{\mathrm{nb}}(\mathrm{t} / \mathrm{h})$, per years of the analyzed period } \\
\hline & & \multicolumn{2}{|c|}{2010} & \multicolumn{2}{|c|}{2011} & \multicolumn{2}{|c|}{2012} & \multicolumn{2}{|c|}{2013} & \multicolumn{2}{|c|}{2014} \\
\hline & & $P_{\mathrm{gb}}(\mathrm{t} / \mathrm{h})$ & $P_{\mathrm{nb}}(\mathrm{t} / \mathrm{h})$ & $P_{\mathrm{gb}}(\mathrm{t} / \mathrm{h})$ & $P_{\mathrm{nb}}(\mathrm{t} / \mathrm{h})$ & $P_{\mathrm{gb}}(\mathrm{t} / \mathrm{h})$ & $P_{\mathrm{nb}}(\mathrm{t} / \mathrm{h})$ & $P_{\mathrm{gb}}(\mathrm{t} / \mathrm{h})$ & $P_{\mathrm{nb}}(\mathrm{t} / \mathrm{h})$ & $P_{\mathrm{gb}}(\mathrm{t} / \mathrm{h})$ & $P_{\mathrm{nb}}(\mathrm{t} / \mathrm{h})$ \\
\hline \multirow{2}{*}{$\mathrm{C} 1$ : cement } & Ship - Silo & 36,2 & 67,4 & 35,2 & 66,1 & 42,1 & 69,5 & 37,3 & 74,7 & 30,9 & 63,1 \\
\hline & $P_{\mathrm{nb}}(\mathrm{t} / \mathrm{h}) / P_{\mathrm{gb}}(\mathrm{t} / \mathrm{h})$ & \multicolumn{2}{|c|}{1,9} & \multicolumn{2}{|c|}{1,9} & \multicolumn{2}{|c|}{1,7} & \multicolumn{2}{|c|}{2,0} & \multicolumn{2}{|c|}{2,0} \\
\hline \multirow[t]{2}{*}{$\mathrm{C} 2$ : Zink ore } & $\begin{array}{c}\text { Open storage area } \\
\text { - ship } \\
\end{array}$ & 215,1 & 517,6 & 221,5 & 455,2 & 313,4 & 465,6 & - & - & 250,7 & 423,5 \\
\hline & $P_{\mathrm{nb}}(\mathrm{t} / \mathrm{h}) / P_{\mathrm{gb}}(\mathrm{t} / \mathrm{h})$ & \multicolumn{2}{|c|}{2,4} & \multicolumn{2}{|c|}{2,1} & \multicolumn{2}{|c|}{1,5} & & \multicolumn{2}{|c|}{1,7} \\
\hline \multirow{2}{*}{ C3: Alumina } & Ship - truck & 18,7 & 66,4 & 61,2 & 74,8 & 57 & 93 & 54,9 & 79,4 & 88,4 & 119,7 \\
\hline & $P_{\mathrm{nb}}(\mathrm{t} / \mathrm{h}) / P_{\mathrm{gb}}(\mathrm{t} / \mathrm{h})$ & \multicolumn{2}{|c|}{3,6} & \multicolumn{2}{|c|}{1,2} & \multicolumn{2}{|c|}{1,6} & \multicolumn{2}{|c|}{1,4} & \multicolumn{2}{|c|}{1,4} \\
\hline \multirow{2}{*}{$\begin{array}{l}\text { C4: Crashed } \\
\text { stone }\end{array}$} & $\begin{array}{c}\text { Open storage area } \\
\text { - ship }\end{array}$ & 33,4 & 109,3 & 28,7 & 101,6 & 99,4 & 200,8 & 92,6 & 159,1 & 87,9 & 147,9 \\
\hline & $P_{\mathrm{nb}}(\mathrm{t} / \mathrm{h}) / P_{\mathrm{gb}}(\mathrm{t} / \mathrm{h})$ & \multicolumn{2}{|c|}{3,3} & \multicolumn{2}{|c|}{3,5} & \multicolumn{2}{|c|}{2,0} & \multicolumn{2}{|c|}{1,7} & \multicolumn{2}{|c|}{1,7} \\
\hline C5: Zinc & $\begin{array}{c}\text { Open storage area } \\
\text { - ship } \\
\end{array}$ & 81,8 & 108,7 & 31,6 & 194,8 & 101,8 & 122,3 & 96,2 & 115,5 & - & - \\
\hline & $P_{\mathrm{nb}}(\mathrm{t} / \mathrm{h}) / P_{\mathrm{gb}}(\mathrm{t} / \mathrm{h})$ & & & & & & & & & & \\
\hline C6: Iron scrap & $\begin{array}{c}\text { Open storage area } \\
\text { - ship } \\
\end{array}$ & 69,3 & 131,6 & 44,2 & 87,5 & 28,8 & 115,2 & 22,4 & 121,8 & 19,8 & 134,8 \\
\hline & $P_{\mathrm{nb}}(\mathrm{t} / \mathrm{h}) / P_{\mathrm{gb}}(\mathrm{t} / \mathrm{h})$ & & & & & & & & & & \\
\hline C7. Grain & Silo - ship & 148,9 & 247 & 55,7 & 226,4 & 78,3 & 227,8 & 140,4 & 210,3 & 97,3 & 214 \\
\hline C. Tradm & $P_{\mathrm{nb}}(\mathrm{t} / \mathrm{h}) / P_{\mathrm{gb}}(\mathrm{t} / \mathrm{h})$ & & & & & & & & & & \\
\hline C8: Salt & Ship - truck & 16,4 & 102,6 & 33,7 & 112 & 46,9 & 104,7 & 52,1 & 152,4 & 80,2 & 131,5 \\
\hline Co. Sall & $P_{\mathrm{nb}}(\mathrm{t} / \mathrm{h}) / P_{\mathrm{gb}}(\mathrm{t} / \mathrm{h})$ & & & & & & & & & & \\
\hline C9. Corn & Silo - ship & - & - & 106,9 & 182,8 & 94,9 & 227,9 & 39,1 & 168,9 & 107,9 & 191,8 \\
\hline C9. Com & $P_{\mathrm{nb}}(\mathrm{t} / \mathrm{h}) / P_{\mathrm{gb}}(\mathrm{t} / \mathrm{h})$ & & & & & & & & & & \\
\hline C10: Coal & Ship - truck & - & - & 110,1 & 192,4 & 105,9 & 147,9 & 97,9 & 113,7 & 132,7 & 196,8 \\
\hline C10.COdI & $P_{\mathrm{nb}}(\mathrm{t} / \mathrm{h}) / P_{\mathrm{gb}}(\mathrm{t} / \mathrm{h})$ & & & & & & & & & & \\
\hline
\end{tabular}

\subsection{Discussion of results}

The biggest variation between the minimal and maximal values of gross berth productivity - $389 \%$ - is registered for handling operation ship to truck with salt and the smallest difference between these two extreme values - $36 \%$ - is characteristic for handling operations ship to silo with cement and handling operation ship to truck with coal.

The biggest variation between the minimal and maximal net berth productivity - $98 \%$ - is registered for handling operation open storage area to ship with crashed stone and the smallest difference between these two extreme values - $17 \%$ - is characteristic for handling operation silo to ship with grain.

Differences between the minimal and maximal values of net berth productivity are smaller than those characteristic for gross berth productivity. This conclusion directly suggests existence of very wide group of influential factors on gross berth productivity of different nature and intensity of influence. It is of crucial importance to recognize those influential factors in order to establish bases for adequate managerial activities directed to reduction of the ship's time in a port.

Differences between the Net berth productivity and Gross berth productivity vary from 20\% (handling operation ship to truck with alumina in the year 2011; handling operation with zinc concentrate for the years 2012 and 2013; handling operation ship to truck with coal in the year 2013) to $580 \%$ for handling operation open storage area to ship with iron scrap in the year 2014.

The narrowest range between the Net berth productivity and gross productivity over the analyzed period is characteristic for handling operation ship to truck with coal (from $20 \%$ in the year 2013 to $70 \%$ in the eyar 2011).

The biggest differences between the values of Net and Gross berth productivity over the considered period 
are registered for handling operation open storage area to ship with iron scrap (from $90 \%$ in the year 2010 to $580 \%$ in the year 2014) and handling operation ship to truck with salt (from $60 \%$ in the year 2014 to $530 \%$ in the year 2010).

When results of analyses are taken into consideration it is necessary to have in mind that the fact that an indicator does not vary intensively over time does not mean that the performance measured by that indicator is necessarily good. It may be consistently bad [13].

\section{Influential factors on berth productivity}

In the available literature are found results of done considerations related to the influential factors on port productivity. Summary of some of the mentioned considerations is given here.

Producing superior productivity is a complex process involving a lot of factors: vessel stowage, vessel size, the volume loaded and discharged, the skills of the crane operators and other dockworkers, the cranes and other assets deployed in working the vessel, and the contractual price the carrier agrees to pay the terminal operator. If yard and gate operations are not operating efficiently, bottlenecks can develop quickly that will affect vessel productivity [7].

One of the major problems that hinders the improvement of productivity is identified as nonoperational times included in the $24 / 7$ operation. Some of the non-operational times during shift are unavoidable e.g. time when operators are checking the oil and water level of the equipment, clean glasses and check for damages before starting the operation, etc. [13].

Set of additional data measuring the likely impact of each factor on output and productivity. From the multitude of such factors the most significant are as follows: size and type of vessels, total tonnage to be discharged/loaded per vessel call, consignment size (average tonnage per $\mathrm{B} / \mathrm{L}$ ), unit size of individual items, packing (type and quality), modal split of cargo movement, working method selected by stevedore and quay cargo handling company (including selection of stevedoring tools), lifting gear and handling equipment used, size of the gang, weather conditions, port of call sequence in a given range, balance (or lack of balance) between various subsystems at a terminal, motivation and quality of terminal personnel, allocation of handling equipment on terminal, place of terminal in port of call sequence, existence or lack of balance between the various subsystems on a terminal [2].

A number of factors affect productivity numbers from port to port and country to country, including the average size of the vessels deployed, whether the port is a gateway port or a transshipment port and the allocation of assets and labor, given the cost of each [12].

It can be concluded that numerous influential factors on port productivity are identified in available literature, but absence of their systematization is evident, as well, some of the important influential factors are not directly recognized.

In further text are presented results of consideration of influential factors on port productivity (with focus on berth productivity). Main groups of influential factors and their principal elements are identified and systematized, based on results of already mentioned analyses found in the available literature and additional researches done by the author.

Group of factors which have decisive influence on port productivity (one of its metrics - berth productivity) are:

- group 1: $F_{1}$ - factors related to port infrastructure: capacity of the port anchorage area; water depth within the port area; number of berths; characteristics of the quay construction; quality of the electric power supply system in the port; characteristics of the port road network; characteristics of the port railway network; model of the port infrastructure maintenance management model; etc.;

- group 2: $F_{2}$ - factors related to port superstructure: characteristics of closed warehouses in the port; characteristics of open storage areas in the port; characteristics of port shore cranes (rail mounted or mobile) - capacity, life cycle phase, technological adequacy, reliability, ...); distances from berths to the port closed warehouses and open storage areas; model of the port superstructure maintenance management model; etc.

- group 3: $F_{3}$ - factors related to port machinery: characteristics of port machinery: capacity, life cycle phase, technological adequacy, reliability, ...; model of the port machinery maintenance management model; etc.

- group 4: $F_{4}$ - factors related to the port organization: port management model; number of port terminal operators; parameters referred on planning, realization and control of working processes; parameters related to port personnel (number, qualifications, ...); etc.

- group 5: $F_{5}$ - characteristics of transport means for transporting cargo to/from the port: characteristics of ships; characteristics of wagons; characteristics of trucks; characteristics of containers;

- $\quad$ group 6: $F_{6}$ - characteristics of cargo handled: type of cargo; characteristics of the manipulation unit; requirements related to the environmental protection; requirements related to the fire protection; requirements related to the safety measures; etc.

- group 7: $F_{7}$ - characteristics of the lifting accessories: type of the lifting accessory, safe working load, etc.;

- group 8: $F_{8}$ - type of the cargo handling operation: ship to shore, ship to wagon, ship to truck, wagon to truck, etc.;

- group 9: $F_{9}$ - factors related to the contract with the customer: contracted terms related to required daily productivity per ship; working and non working hours; etc.

- group 10: $F_{10}$ - factors related to the port information system: model of collecting, analyzing and distributing data related to the cargo handling process; etc.;

- group 11: $F_{11}$ - factors related to necessary administrative procedures: customs procedures, state inspections controls, etc.;

- group 12: $F_{12}$ - factors related to the climate in the port zone: temperatures, winds, humidity, etc.; 
- group 13: $F_{13}$ - factors related ot the position of the port in the supply chain: port of call sequence, etc.;

- group 14: $F_{14}$ - factors related to the adequacy level of the port (terminal) composition: if subsystems are well ballanced; etc.

\section{Conclusion}

By the consideration done in this paper is clearly confirmed starting hypothesis of the conducted research about existence of significant differences between Net and Gross berth productivity (about significant share of non operational time in the total berthing time).

Values of berth productivity are determined by numerous groups of factors of very different nature and intensity of influence, which are identified and systematized.

In order to create a model for optimizing berth productivity (to contribute to the maximal reduction of the ship turnaround time), intention of the authors is to continue researches of correlations between the berth productivity values and identified factors of influence.

\section{References}

[1] Esmer, S. Performance Measurements of Container Terminal Operations, Dokuz Eylul Universitesi, 2008. http://www.sbe.deu.edu.tr/dergi/cilt10.say\%C4\%B11/10.1 \%20esmer.pdf (07.11.2014)

[2] De Monie, G. Measuring and Evaluating Port Performance and Productivity. UNCTAD Monographs on Port Management. http://unctad.org/en/Docs/ship4946 en.pdf (14.10.2014)

[3] Improving Marine Container Terminal Productivity: Development of productivity measures, proposed sources of data, and initial collection of data from proposed sources. The Tioga Group Inc. 2010. http://www.marad.dot.gov/ documents/070810_Tioga_CHCP_Productivity_Report.pdf $(23.09 .2014)$

[4] Port performance indicators. UNCTAD. United Nations (TD/B/C4/131/Supp.1/Rev.1).

[5] Wang, T. F.; Song, D. W.; Cullinane, K. The Applicability of Data Envelopment Analysis to Efficiency Measurement of Container Ports. http://www.cepal.org/transporte/ perfil/iame_papers/.../Wang_et_al.doc (22.08.2014)

[6] Merk, O.; Dang, T. Efficiency of world ports in container and bulk cargo (oil, coal, ores and grain). OECD Regional Development Working Papers. 2012. http://www.oecdilibrary.org/docserver/download/5k92vgw39zs2.pdf? expires $=1420669707 \& \mathrm{id}=\mathrm{id} \&$ accname $=$ guest $\&$ checksum $=$ 0B81F49E950541D4BB03D2D6FD86E493 (21.11.2014)

[7] Key Findings on Terminal Productivity Performance Across Ports, Countries and Regions. JOC Group. 2013. http://www.portoflosangeles.org/Board/2013/September\%2 02013/091913_Agenda_Audit_Committee_Item_3.pdf (14.12.2014)

[8] http://www.investorwords.com/3876/productivity.html (12.07.2014)

[9] The Handbook of Maritime Economics and Business. // Revisiting the Productivity and Efficiency of Ports and Terminals: Methods and Applications / Kevin Cullinane. London : Lloyd's List, 2010. pp. 907-929.

[10] Kasypi, M.; Shan, M. Z. A Productivity Analysis of Medium Seaport Container Terminal. 2011. http://www.sustainability.utm.my/cipd/files/2011/10/A-
Productivity-Analysis-of-Medium-Seaport-ContainerTerminal.pdf (01.12.2014)

[11] http://www.joc.com/special-topics/port-productivity (14.07.2014)

[12] Berth Productivity. The Trends, Outlook and Market Forces Impacting Ship Turnaround Times. JOC Group. 2014 http://www.knowledgeplanning.org/upload/news/JOC-PPwhitepaper-v\%20(1).pdf (15.11.2014)

[13] Shanthirathne, A. How to Improve My Port's Productivity/Efficiency? Sri Lanka Ports Authority. http://www.iaphworldports.org/LinkClick.aspx?fileticket= UOaW S0HbUQ\%3D\&tabid=5033 (29.11.2014)

[14] Ilmer, $\bar{M}$. Enhancing container terminal productivity: A comaker approach between carriers and operators. http://www.porttechnology.org/images/uploads/technical_p apers/PT26-14.pdf (23.10.2014)

[15] Beresdorf, A. K. C. et al. UNCTAD and WORKPORT models of port development: evolution or revolution. // Maritime Policy and Management. 31, 2(2004), pp. 93-107. https://doi.org/10.1080/0308883042000205061

[16] Documentation of the Port of Bar. Bar. 2014.

[17] Vukadinovic, S. Elements of the theory of probability and mathematical statistics, Privredni pregled, Beograd, 1986.

\section{Authors' addresses:}

Dr. Sc. Deda Đelović, Director of the Development Department Port of Bar

Obala 13. jula b.b.

85000 Bar, Montenegro

E-mail: deda.djelovic@lukabar.me

Dr. Sc. Dijana Medenica Mitrović, Assistant Professor Faculty for Business Management

Ul. Marsala Tita 7

85000 Bar, Montenegro

E-mail: disan@t-com.me 